\title{
Conflitos Familiares e Práticas Educativas Parentais como Preditores de Dependência de Internet
}

\author{
Michele Terres-Trindade - Universidade do Vale do Rio dos Sinos, São Leopoldo, Brasil \\ Clarisse Pereira Mosmann - Universidade do Vale do Rio dos Sinos, São Leopoldo, Brasil
}

\begin{abstract}
Resumo
Esta pesquisa objetivou analisar o efeito preditor dos motivos de conflito entre pais e filhos, do conflito interparental e das práticas educativas parentais para a dependência de internet (DI) em jovens. A amostra foi constituída por 200 indivíduos (152 meninas e 48 meninos), com idades de 15 a 24 anos, 85,5\% residentes no Rio Grande do Sul e 14,5\% em outros estados brasileiros. Os participantes responderam individualmente ao protocolo disponível on-line. Os resultados indicaram como preditores os conflitos sobre a internet com o pai, a ameaça do conflito interparental, os conflitos sobre internet com a mãe e a prática de apoio emocional paterno. Juntas, essas variáveis explicaram $21,2 \%$ da dependência de internet. Os resultados corroboram estudos internacionais e indicam que as variáveis familiares investigadas têm um efeito considerável na predição da DI, tendo em vista que esse é um fenômeno complexo e de natureza multifatorial.

Palavras-chave: internet (dependência), estilo parental, relações pais-criança, conflito conjugal
\end{abstract}

\section{Family Conflict and Parenting Practices as Predictors of Internet Addiction}

\begin{abstract}
This research aimed to analyze the predictive effect of reasons for conflict between parents and children, the interparental conflict and parenting practices for Internet addiction (IA) in young people. The sample consisted of 200 people (152 girls and 48 boys), between 15 and 24 years of age, 85.5\% residing in Rio Grande do Sul and 14.5\% in other states. Participants individually responded to the protocol available online. Results indicated as predictors conflicts over the internet with the father, the threat of interparental conflict, conflict over the internet with the mother and the practice of parental emotional support. Together, these variables explained $21.2 \%$ of Internet addiction. The results corroborate with international studies indicating that family variables investigated have a significant role in predicting the IA, considering that this is a complex and multifactorial phenomenon.
\end{abstract}

Keywords: internet (addiction), parenting, parent-child relations, family conflict

\section{Conflictos Familiares y Prácticas Educativas Parentales como Predictores de la Dependencia de Internet}

\begin{abstract}
Resumen
Esta investigación se dedicó a examinar el efecto predictor de los motivos de conflicto entre padres e hijos, del conflicto entre los padres y de las prácticas educativas parentales en la dependencia a Internet (DI) de jóvenes. Participaron de la muestra 200 individuos (152 de sexo femenino y 48 de sexo masculino), de 15 a 24 años de edad, 85,5\% residentes en Río Grande del Sur y el 14,5\% en otros estados brasileños. Los participantes respondieron individualmente al protocolo disponible on-line. Los resultados indicaron como predictores; los conflictos con el padre sobre la Internet, la amenaza del conflicto entre los padres, los conflictos sobre Internet con la madre y la práctica de apoyo emocional paterno. Juntas, esas variables explicaron $21,2 \%$ de la dependencia de Internet. Los resultados corroboran estudios internacionales e indican que las variables familiares investigadas tienen un efecto considerable en la predicción de la DI, teniendo en cuenta que ese es un fenómeno complejo y de naturaleza multifactorial.

Palabras-clave: internet (dependencia), estilo parental, relaciones padres-niños, conflicto conjugal
\end{abstract}

Com o número cada vez maior de dispositivos tecnológicos passíveis de conexão com a internet, o acesso à rede mundial de computadores se mostra em crescimento desde a sua popularização na década de 1990. Nos últimos anos, a internet deixou de ser uma ferramenta utilizada apenas para fins acadêmicos ou profissionais, tornando-se parte do cotidiano das pessoas em diversos momentos. A população jovem tem se destacado quando o assunto é o acesso ao universo on-line, especialmente, para uso não essencial (quando o acesso à internet não está relacionado ao trabalho ou estudo, por exemplo). No Brasil, uma pesquisa realizada pelo Núcleo de Informação e Coordenação do Ponto BR no ano de 2005, apontou que 42,18\% dos jovens brasileiros residentes na área urbana, com idade de 16 a 24 anos, declaravam acessar a internet diariamente; em 2012 o percentual saltou para 68\% (Núcleo de Informação e Coordenação do Ponto BR [NICBR], 2012).

Outra pesquisa brasileira realizada pelo Centro de Estudos sobre as Tecnologias da Informação 
e Comunicação [CETIC] (2012) com o objetivo de medir os hábitos de uso da população com acesso à internet, com idade entre nove e 16 anos, envolveu 1.580 crianças/adolescentes e o mesmo número de pais/ responsáveis. Os resultados mostraram que, embora o acesso à rede tenha aumentado consideravelmente, os pais têm pouco ou nenhum conhecimento do que os filhos fazem quando estão conectados. Especificamente, quando questionados sobre o grau de conhecimento dos pais acerca das suas atividades na internet, 27\% dos participantes revelaram "muito", 41\% "mais ou menos", 19\% "apenas um pouco" e 13\% "nada" (CETIC, 2012). Dessa forma, o aumento do uso da internet associado à percepção da pouca supervisão dos pais por parte dos adolescentes suscita questionamentos: quando o uso da internet pode ser considerado prejudicial? Os pais podem prevenir o uso excessivo da internet? De que forma? Essas indagações indicam a necessidade de realização de pesquisas que contemplem a temática do uso da internet entre jovens, investigando de que forma a dinâmica familiar se expressa na interação com essa peculiaridade nessa nova geração.

Buscando respostas para essas perguntas, são encontrados na literatura diversos trabalhos relatando os problemas relacionados ao uso da internet, apontando que o uso prejudicial dessa tecnologia vai muito além do número de horas de conexão. Os estudos indicam que entre os comportamentos descritos como características da dependência de internet (DI) estão, especialmente, a preocupação excessiva ou descontrolada do indivíduo para manter-se conectado à rede, associada ao sofrimento ou a prejuízos em atividades escolares, sociais, ocupacionais, entre outros (Young \& Abreu, 2011).

Em meados dos anos 1990, começaram a ser apresentados os primeiros estudos acerca do uso prejudicial da internet (Young, 1996, 1998) e, desde então, inúmeras investigações vêm sendo conduzidas buscando compreender e caracterizar essa possível nova condição psicopatológica. No entanto, até hoje a DI ainda apresenta critérios diagnósticos diversificados, além de não estar classificada como um quadro clínico psiquiátrico em nenhum manual diagnóstico. Não obstante, o crescente uso da internet e os problemas associados aos usuários fizeram com que as pesquisas continuassem avançando mesmo diante das divergências diagnósticas, especialmente em países do Extremo Oriente onde o progresso tecnológico ocorre mais rapidamente (Park, Kim, \& Cho, 2008; Yu \& Shek, 2013). Teoricamente, a DI é conceituada como um transtorno complexo, de natureza multifatorial, que integra o espectro compulsivo-impulsivo e é caracterizada, principalmente, por um baixo controle do uso da internet (Dell'Osso, Altamura, Allen, Marazziti, \& Hollander, 2006). Sendo assim, embora o indivíduo reconheça os prejuízos associados ao uso excessivo da internet, ele continua mantendo as atividades on-line de maneira recorrente e persistente.

Além do termo "dependência de internet", outras denominações, como uso excessivo de internet, uso problemático de internet e transtorno por uso de internet são empregadas para designar essa condição (Abreu, Karam, Góes, \& Spritzer, 2008). Entretanto, longe de haver consenso, o termo "internet addiction" (dependência de internet) é mais comumente utilizado na literatura sobre esse tema e, sendo assim, foi adotado no presente estudo (Ak, Koruklu, \& Y1lmaz, 2013; Lam, Peng, Mai, \& Jing, 2009; Park et al., 2008; Yen, Yen, Chen, Chen, $\&$ Ko, 2007).

Entre os estudos internacionais produzidos sobre DI, encontram-se diferentes resultados de prevalência, apontando índices que variam de 1,5\% e 8,2\% na Europa e Estados Unidos e de 5,52\% e 20,3\% em jovens de países do Extremo Oriente (Weinstein \& Lejoyeux, 2010). Esses diferentes percentuais se devem ao fato de que existem distintos critérios diagnósticos e instrumentos de medida variados sendo utilizados para avaliar a DI. Outros achados apontam que, entre a população que possui computador com acesso à internet, os adolescentes e os adultos jovens são os mais vulneráveis (Young \& Abreu, 2011; Fortson, Scotti, Chen, Malone, \& Del Ben, 2007).

Enquanto alguns pesquisadores internacionais tentam identificar a prevalência, os critérios diagnósticos e os fatores pessoais envolvidos na DI, em países do Extremo Oriente, em especial, as investigações avançam, buscando identificar as variáveis familiares envolvidas, a fim de que ações de prevenção possam ser desenvolvidas diante dos elevados índices de dependência entre a população jovem desses países. Entre as variáveis que envolvem a família, os estudos apontam como preditores: a insatisfação familiar (Lam et al., 2009), os altos níveis de conflito interparental e entre pais-filhos, bem como práticas educativas parentais punitivas (Li, Garland, \& Howard, 2014; Yen et al., 2007). Além disso, os estudos assinalam que as variáveis familiares, como os conflitos e coesão familiar, podem atuar como fatores de proteção ou risco para a DI entre os jovens (Park et al., 2008).

Nesse sentido, é consenso na literatura que a família está intimamente relacionada aos problemas 
emocionais e de comportamento expressos na infância e na adolescência (Erel \& Burman, 1995; Villas-Boas, Dessen, \& Melchiori, 2010). Elevados níveis de conflito familiar, aliados às práticas educativas parentais negativas estão associados a problemas internalizantes e externalizantes em crianças e adolescentes (Benetti, 2006; Bernal, 2012; Bolsoni-Silva, Paiva, \& Barbosa, 2009; Goulart, Wagner, \& Mosmann, 2014; Linares, Rusillo, Cruz, \& Fernández, 2011). Sendo assim, uma vez que a DI é considerada um transtorno do espectro compulsivo-impulsivo e, portanto, um problema externalizante, torna-se pertinente compreender $\mathrm{O}$ fenômeno a partir dessa perspectiva, buscando investigar quais variáveis familiares podem estar associadas a DI em jovens brasileiros.

Os conflitos familiares podem ser divididos em conflito pais-filho e conflito conjugal. Entre pais e filhos, os conflitos são comuns na fase da adolescência e adulta jovem e podem ocorrer por diversos motivos, principalmente àqueles vinculados ao cotidiano familiar (Bernal, 2012). Em geral, a mãe ainda é a maior responsável pelas atividades ligadas à família (Wagner, Falcke, Silveira, \& Mosmann, 2002; Wagner, Predebon, Mosmann, \& Verza, 2005) e, por consequência, os conflitos com ela podem ser mais frequentes. Além disso, existem diferenças entre pai e mãe no relacionamento com os filhos. Os pais estão, tradicionalmente, mais voltados à proteção externa, proporcionando uma visão do mundo aos filhos, enquanto as mães estão mais ligadas à proteção dentro do lar, mediando as relações entre os membros da família (Bögels \& Perotti, 2014), o que pode explicar os contrastes nos conflitos pai-filho e mãe-filho. Já o conflito conjugal - quando investigado a partir da perspectiva dos filhos é denominado de conflito interparental - atinge de forma direta a relação pais e filhos, conforme sustenta a hipótese de spillover (Erel \& Burman, 1995; Mosmann, Wagner, \& Sarriera, 2008). Nesse entendimento, a relação conjugal conflituosa transborda e influencia negativamente a relação com os filhos, contribuindo para um ambiente familiar permeado por discórdias e promovendo a manifestação de sintomas nos filhos.

O funcionamento conjugal repercute na relação parental, expresso nas práticas educativas parentais, as quais estão, de acordo com vasta literatura nacional e internacional, associadas aos problemas de ajustamento na infância e adolescência (Bolsoni-Silva et al., 2009; Buehler \& Gerard, 2002; Linares et al., 2011). As denominadas práticas educativas positivas, caracterizadas por maior proximidade e responsividade, correlacionam-se negativamente com os problemas externalizantes e atuam de forma protetiva contra esses problemas. Já as práticas educativas negativas, caracterizam-se por atitudes mais coercitivas, apresentam correlações positivas e mostram-se como fatores de risco para os problemas externalizantes (Bolsoni-Silva et al., 2009; Linares et al., 2011; Mosmann, Costa, Luz, \& Silva, 2014).

Sendo assim, ainda que as investigações sobre a DI e as relações familiares tenham apresentado avanços na literatura internacional, percebe-se que algumas das especificidades dessas interações ainda não foram esclarecidas. Os estudos atuais denotam não considerar as particularidades do papel do pai e da mãe na família quando da investigação dos conflitos entre pais e filhos e das práticas educativas parentais, uma vez que essas variáveis não são avaliadas separadamente na predição da DI. Ademais, a literatura ainda não aponta especificamente quais são os conflitos familiares relacionados com a DI, nem o seu poder de predição. Outra lacuna é a carência de estudos que avaliem conjuntamente as variáveis familiares de conflitos pais-filho, conflitos conjugais e práticas educativas parentais como preditores de DI e, portanto, ainda não está bem estabelecido se alguma dessas variáveis prepondera na predição de DI em jovens.

Tendo em vista que a literatura aponta a população jovem como a mais atingida pela DI (Young \& Abreu, 2011), bem como se encontram indícios de que as variáveis familiares se expressam na DI em jovens de outros países (Lam et al., 2009; Li et al., 2014; Yen et al., 2007), identifica-se uma lacuna nas investigações nacionais que não versam sobre a temática das relações familiares e suas repercussões na DI. Por conseguinte, isso justifica a realização do presente estudo, cujo objetivo foi analisar o efeito preditor dos motivos de conflito entre pais e filhos, das práticas educativas parentais e do conflito interparental para a dependência de internet em uma amostra de jovens brasileiros.

\section{Método}

\section{Delineamento}

Trata-se de um estudo explicativo, quantitativo e de corte transversal.

\section{Amostra}

Esta investigação contou com 200 jovens com idades de 15 a 24 anos $(M=20,24 ; D P=2,77)$ que moravam, na companhia de, pelo menos, um dos pais. Destes, $85,5 \%(n=171)$ eram residentes no estado do 
Rio Grande do Sul e 14,5\% ( $n=29)$ em outros 12 estados do Brasil.

\section{Instrumentos}

Os jovens responderam a um protocolo constituído por cinco partes:

Parte I - Questionário Sociobiodemográfico. Informações relativas à idade, sexo, escolaridade, configuração familiar e classe social percebida (Liu, Ali, Soleck, Hopps, \& Pickett Jr., 2004), etc.

Parte II - Escala de Conflito Pais-filho (ECPF). Originalmente desenvolvida por (Buehler \& Gerard, 2002) (adaptada por Mosmann, 2007) para avaliar o conflito conjugal. Nesse estudo, o instrumento foi adaptado para avaliar os motivos de conflito dos jovens com seus pais, respondido separadamente para pai e mãe. Os itens referentes aos motivos de conflito incluíram: tarefas domésticas, escola, amizades/ namoro, internet, drogas e dinheiro. A escala constitui-se de seis itens medidos em uma escala Likert de seis pontos, variando de $1=$ quase nunca até $6=$ quase todos os dias. Maiores escores na escala representam altos níveis de conflito (Buehler \& Gerard, 2002). O coeficiente alpha de Cronbach obtido para a ECPF total neste estudo foi de $\alpha=0,75$.

Parte III - Escala de Práticas Parentais (EPP). Desenvolvida por Teixeira, Oliveira e Wottrich (2006), a escala busca identificar as práticas educativas parentais por meio da percepção dos filhos e é composta por 27 itens e seis dimensões: apoio emocional, controle punitivo, incentivo à autonomia, intrusividade, supervisão do comportamento e cobrança de responsabilidade, as quais são medidas em uma escala Likert de cinco pontos, com definições-âncora nos extremos da escala $(1=$ quase nunca ou bem pouco e 5 = geralmente ou bastante). Os índices de consistência interna encontrados no estudo original foram alpha de Cronbach 0,89 (apoio emocional), 0,73 (controle punitivo), 0,76 (incentivo à autonomia), 0,67 (intrusividade), 0,77 (supervisão do comportamento) e 0,70 (cobrança de responsabilidade) (Teixeira et al., 2006). Neste estudo, o coeficiente alpha de Cronbach obtido para a EPP total foi de 0,93.

Parte IV - Escala de Percepção dos Filhos sobre Conflito Interparental (EPFCI). EPFCI (Childrens' Perception of Interparental Conflict Scale, Grych, Seid, \& Fincham, 1992) traduzida e adaptada por Wagner (2010), foi desenvolvida para avaliar as opiniões dos filhos sobre diversos aspectos do conflito conjugal de seus pais. A escala é constituída por 50 itens, medidos apor meio de uma escala Likert de cinco pontos, com definições-âncora variando de $1=$ totalmente verdadeiro a 5 totalmente falso, e subdivididos em nove subescalas, sendo quatro delas para descrever as dimensões dos conflitos conjugais (frequência, intensidade, resolução e conteúdo) percebidos pelos filhos. Outras quatro subescalas descrevem a reação ou a interpretação dos filhos diante dos conflitos (culpabilidade, sensação de ameaça, eficácia nas estratégias de enfrentamento e percepção da estabilidade da causa do conflito). E a última subescala, nomeada de triangulação, refere-se à probabilidade de envolvimento do filho no conflito conjugal. O coeficiente alpha de Cronbach obtido para a EPFCI neste estudo foi de 0,94 .

Parte V - Teste de Dependência de Internet (IAT). O Internet Addiction Test - IAT (Young, 1998 - traduzido e adaptado por Conti, et al., 2012) é considerado o primeiro instrumento para a avaliação da dependência de internet e encontra-se adaptado para diversos idiomas, incluindo o português (Conti, et al., 2012; Jelenchick, Megan, \& Moreno, 2012; Widyanto \& McMurran, 2004). É autoadministrado, formado por 20 itens, que medem a extensão do envolvimento com o computador e classifica a dependência em termos de prejuízo, incluindo o grau em que o uso da internet afeta a rotina diária, a vida social, a produtividade e o padrão de sono do indivíduo (Conti, et al., 2012; Young \& Abreu, 2011; Widyanto \& McMurran, 2004).

Os respondentes devem classificar os itens em uma escala Likert de cinco pontos (1 = "raramente", 2 = "ocasionalmente", 3 = "frequentemente", 4 = "geralmente" e 5 = "sempre"). A pontuação mínima do teste é 20, e a máxima é de 100 de pontos; quanto maior a pontuação, mais severa é a dependência. Sendo assim, o instrumento pode classificar a DI em três níveis: usuário com controle, usuário com problemas e usuário grave. A análise de consistência interna da versão em português do instrumento foi de $\alpha=0,85$ (Conti, et al., 2012) e neste estudo o coeficiente alpha de Cronbach foi de $\alpha=0,87$.

\section{Procedimentos de Coleta de Dados}

A coleta de dados foi realizada no período de março a junho de 2013. Para o acesso à amostra foram utilizados dois métodos, realizados de acordo com a idade dos participantes. Para a seleção dos maiores de idade (com 18 anos ou mais), utilizou-se a técnica snowball sampling (bola de neve) e, neste estudo, se deu por intermédio de convites por e-mail e redes sociais. $\mathrm{O}$ acesso aos menores de idade (15 a 17 anos) seguiu o 
critério de conveniência, por meio de convites através de escolas da rede pública e privada da região metropolitana de Porto Alegre/RS.

A fim de coletar os dados dos participantes maiores de 18 anos, um link para o site da internet com informações sobre a pesquisa foi divulgado por e-mail e, igualmente, pela rede social Facebook (www.facebook. com) através da lista de contatos da pesquisadora. Por meio do compartilhamento do link da pesquisa por diversos usuários da rede social, o efeito bola de neve foi alcançado.

Ao clicarem no link disponibilizado on-line, os participantes tinham acesso à apresentação da pesquisa, informações acerca dos aspectos éticos, dos critérios de inclusão (ser maior de idade e residir com, pelo menos, um dos pais) e o modo de participação na pesquisa. Os usuários que aceitavam participar do estudo eram orientados a confirmar sua participação informando um endereço eletrônico. Na tela seguinte, os participantes se deparavam com o formulário eletrônico contendo as questões do instrumento de pesquisa. As respostas enviadas foram armazenadas eletronicamente em um servidor de internet, protegido por senha, juntamente com as informações de data e hora do preenchimento do instrumento.

$\mathrm{Na}$ coleta de dados dos menores de idade, a pesquisadora contatou a direção das escolas apresentando a pesquisa, os objetivos e o modo de participação dos alunos-alvo. Após a aceitação da escola, as turmas eram visitadas e os alunos convidados a participarem do estudo. Aqueles que aceitavam e estavam de acordo com os critérios de inclusão (idade de 15 a 17 anos e residiam com, pelo menos, um dos pais), eram orientados a solicitar a assinatura do Termo de Consentimento Livre e Esclarecido (TCLE) por um dos responsáveis. Somente aqueles que devolviam o TCLE assinado, recebiam uma senha e o endereço do site para responderem o instrumento disponível on-line.

O site para coleta de dados dos menores de idade era idêntico ao dos participantes maiores de idade, excetuando-se o campo senha que foi adicionado, com o objetivo de que apenas os alunos que receberam uma senha pudessem participar do estudo. Dezessete participantes que responderam o instrumento, mas não estavam de acordo com os critérios de inclusão deste estudo foram excluídos do banco de dados.

\section{Considerações Éticas}

Este estudo foi aprovado pelo Comitê de Ética em Pesquisa sob protocolo número 187.637 e seguiu todas as diretrizes e normas regulamentadoras de pesquisas envolvendo seres humanos.

\section{Análise de Dados}

Foram realizadas análises descritivas para observar o comportamento das variáveis por meio de frequências, médias e desvio padrão. Com o objetivo de investigar como se associavam as variáveis dos motivos conflitos com os pais, as práticas educativas parentais, o conflito interparental e o IAT, foi realizado o Teste de Correlação de Pearson.

Uma análise de regressão múltipla foi realizada com o objetivo de analisar os motivos de conflito, as práticas educativas e o conflito interparental como conjunto de variáveis preditoras para a dependência de internet. As análises estatísticas foram realizadas no programa SPSS (Statistical Package for the Social Sciences), versão 17 e o nível de significância adotado foi de 0,05 $(p>0,05)$.

\section{Resultados}

$\mathrm{Na}$ Tabela 1 pode-se observar uma descrição sumária das características dos participantes do presente estudo.

A maior parte da amostra foi composta por participantes do sexo feminino, com escolaridade de nível superior, que está trabalhando e pertence a famílias nucleares (formadas por pai-mãe-filhos). Quanto à classe social percebida, caracterizada por quando o próprio participante declara a qual classe social ele acredita que pertence, predominaram os participantes da classe média.

Pode-se observar que as tarefas domésticas apresentam maior média de conflito dos jovens com a mãe, seguido pelos desentendimentos sobre dinheiro e internet. Com o pai, os conflitos sobre o dinheiro têm maior média do que em relação às tarefas domésticas e a internet também é apontada como terceiro motivo de discórdia entre os participantes e seu pai.

A pontuação média dos participantes no IAT foi de 23 pontos $(D P=11,97)$, sendo $89,0 \%(n=178)$ classificados como usuário com controle do uso de internet e 11,0\% $(n=22)$ como usuário com problemas devido ao uso da internet, conforme pontos de corte do instrumento (Widyanto \& McMurran, 2004). Não houve participantes classificados na categoria usuário grave na amostra estudada.

Foram encontradas associações estatisticamente significativas entre o escore total do IAT e as dimensões 
Tabela 1

Caracterização dos Participantes

\begin{tabular}{ll}
\hline Caracterização dos participantes & \\
\hline Variáveis & $n(\%)$ \\
\hline Sexo & $152(76,0 \%)$ \\
Feminino & $48(24,0 \%)$ \\
Masculino & \\
Escolaridade & $51(25,5 \%)$ \\
Ensino médio & $136(68,0 \%)$ \\
Graduação & $13(6,5 \%)$ \\
Pós-graduação & \\
Está trabalhando atualmente & $111(55,5 \%)$ \\
Sim & $89(44,5 \%)$ \\
Não & \\
Classe social percebida & $52(26,0 \%)$ \\
Média baixa & $96(48,0 \%)$ \\
Média & $52(26,0 \%)$ \\
Média alta & $12(6,0 \%)$ \\
Configuração familiar & $137(68,5 \%)$ \\
Nuclear & $37(18,5 \%)$ \\
Monoparental & $14(7,0 \%)$ \\
Família extensa & \\
Reconstituída & \\
\hline
\end{tabular}

Tabela 2

Motivos de Conflito dos Jovens com os Pais

\begin{tabular}{lcccc}
\hline Motivos de conflito dos jovens com os pais \\
\hline \multirow{2}{*}{ Motivos } & \multicolumn{2}{c}{ Mãe } & \multicolumn{2}{c}{ Pai } \\
\cline { 2 - 6 } & \multicolumn{3}{c}{ DP } & \multicolumn{2}{c}{ DP } \\
\hline Tarefas domésticas & 2,89 & 1,72 & 1,80 & 1,27 \\
Dinheiro & 2,07 & 1,38 & 1,85 & 1,28 \\
Internet & 1,85 & 1,30 & 1,65 & 1,15 \\
Amizades/namoro & 1,60 & 1,01 & 1,43 & 0,90 \\
Estudos & 1,48 & 0,99 & 1,45 & 0,96 \\
Drogas & 1,08 & 0,48 & 1,07 & 0,42 \\
\hline
\end{tabular}

avaliadas pelas escalas, especificamente, associações relacionadas de forma negativa com as práticas educativas de apoio emocional materno $(p=0,036 ; r=-0,148)$ e paterno $(p=0,019 ; r=-166)$ e de incentivo a autonomia paterna $(\phi=0,011 ; r=-0,179)$. Os escores do IAT apresentaram correlações positivas com os conflitos com a mãe sobre internet $(p<0,001 ; r=0,334)$ e os conflitos com o pai sobre: internet $(p<0,001 ; r=0,357)$, tarefas domésticas $(p=0,002 ; r=0,220)$ e dinheiro $(p$ $=0,011 ; r=0,180)$. Além de relações positivas com as práticas parentais de intrusividade materna $(p=0,050$; $r=0,139)$ e paterna $(\phi=0,006 ; r=0,194)$ e controle punitivo paterno $(p=0,020 ; r=0,164)$, supervisão do comportamento paterno $(\phi=0,043 ; r=0,143)$. Em relação à percepção do conflito interparental, o IAT apresentou relações positivas com as dimensões de conteúdo $(p=0,017 ; r=0,169)$, sensação de ameaça $(p<0,001 ; r=0,246)$ e culpabilidade $(\phi=0,042 ; r=$ $0,144)$.

$\mathrm{Na}$ análise de regressão linear múltipla, foram inseridas como variáveis independentes apenas aquelas que apresentaram correlações significativas com o IAT. Foram testados quatro modelos, sendo este último (Modelo 4) o que indicou um conjunto das variáveis preditoras capaz de explicar $21,2 \%$ da dependência de internet dos participantes deste estudo, confirme indica a Tabela 3.

O modelo final apresentou a associação entre as variáveis de critério e explicativas moderada (Dancey \& Reidy, 2006). Juntos, os conflitos sobre a internet com o pai, a ameaça do conflito interparental e os conflitos sobre internet com a mãe estão positivamente relacionados com a dependência de internet, enquanto a prática de apoio emocional paterno está correlacionada negativamente. Os coeficientes de regressão padronizados indicam que o conflito sobre internet $\operatorname{com}$ o pai $(\beta=$ $0,357)$ é preditor mais robusto do que a ameaça do conflito interparental $(\beta=0,192)$ e que o conflito sobre internet com a mãe $(\beta=0,177)$. Todavia, essas variáveis estão positiva e significativamente relacionadas à dependência de internet, enquanto a prática de apoio emocional paterno $(\beta=-0,155)$ está negativa e significativamente relacionada.

\section{Discussão}

Este estudo apresentou como objetivo analisar o efeito preditor dos motivos de conflito entre pais e filhos, do conflito interparental e das práticas educativas parentais para a dependência de internet em uma 
Tabela 3

Preditores de Dependência de Internet

Preditores de Dependência de Internet

\begin{tabular}{lcccccc}
\hline & \multicolumn{5}{c}{ Dependência de Internet } \\
\cline { 2 - 7 } & Mod. 1 & Mod. 2 & Mod. 3 & \multicolumn{2}{c}{ Modelo 4 } \\
\hline Preditores & B & B & B & B & EP & $\beta$ \\
Conflitos internet [pai] & 3,730 & 3,454 & 3,381 & 2,349 & 0,693 & 0,357 \\
Ameaça conflito interparental & & 0,422 & 0,426 & 0,400 & 0,134 & 0,192 \\
Prática apoio emocional [pai] & & & $-0,210$ & $-0,214$ & 0,088 & $-0,155$ \\
Conflitos internet [mãe] & & & & 1,621 & 0,712 & 0,177 \\
R & 0,357 & 0,410 & 0,437 & & 0,461 & \\
$R^{2}$ & 0,128 & 0,168 & 0,191 & & 0,212 & \\
$R^{2}$ Ajustado & 0,123 & 0,160 & 0,179 & & 0,196 & \\
Durbin-Watson & & & & & & \\
\hline
\end{tabular}

Notas. $\mathrm{N}=200$. Mod. $=$ modelo $\mathrm{EP}=$ erro padrão.

amostra de jovens brasileiros. Os resultados mostraram que a internet aparece como motivo de conflito com os pais na amostra estudada, sendo menos frequente apenas do que os conflitos sobre atividades do cotidiano, como as tarefas domésticas e o dinheiro (Bernal, 2012). Os conflitos com a mãe sobre todas as temáticas mostram-se mais frequentes quando comparados com o pai, o que pode estar relacionado ao fato de que ainda as mães estão mais presentes no dia a dia dos filhos em comparação aos pais (Wagner et al., 2002; Wagner et al., 2005).

Quanto à classificação dos participantes em relação ao nível de dependência, os índices encontrados na amostra brasileira são muito similares aos de um estudo realizado com jovens chineses utilizando o mesmo instrumento, onde $89,2 \%$ dos participantes foram classificados como usuários com controle, 10,2\% usuários com problemas e, por fim, 0,6\% dependentes (Lam et al., 2009). Chama a atenção que os resultados são semelhantes, apesar das diferenças culturais existentes entre os dois países, além do fato de que os avanços tecnológicos são muito maiores e o acesso à internet é amplamente difundido quando se compara a China à realidade brasileira.

As associações encontradas entre as práticas educativas parentais e os níveis de DI são fracas. No entanto, pode-se considerar que esse resultado se deve ao fato de não haver classificação de participantes com DI grave, bem como média de pontuação no IAT ser considerada baixa. Não obstante, compreendendo-se a DI como um sintoma externalizante, as correlações encontradas refletem o que é visto na literatura sobre essa forma de expressão dos sintomas dos filhos (Bolsoni-Silva et al., 2009; Linares et al., 2011; Mosmann et al., 2014). Os resultados apontam as práticas educativas positivas de apoio emocional materno e paterno, incentivo à autonomia materno e paterno e a supervisão do comportamento paterno como um fator de proteção para a DI. Em contrapartida, as práticas educativas negativas de intrusividade materna e paterna e controle punitivo paterno, são um fator de risco. Chama a atenção a prática de supervisão do comportamento que, de acordo com o estudo original (Teixeira et al., 2006), seria considerada positiva, mas neste estudo caracterizou-se como um fator de risco para a DI. Tendo em vista a idade média dos participantes, pode-se supor que a supervisão exercida pelo pai estaria sendo interpretada como controle por parte dos filhos. Além disso, essa supervisão pode indicar uma menor habilidade do pai em lidar com os problemas do filho que, por isso, utiliza-se de práticas mais coercitivas. Esse comportamento do pai poderia explicar porque entre os conflitos entre pais e filhos encontrados neste estudo mais relacionados à DI são aqueles com o pai sobre internet, tarefas domésticas e dinheiro.

A análise de regressão permitiu identificar, neste estudo, um conjunto de variáveis familiares capaz de explicar $21,2 \%$ da DI em jovens brasileiros, mesmo 
diante da complexidade de mensuração e explicação de um fenômeno de natureza multifatorial. Entre as variáveis investigadas, destaca-se o efeito dos conflitos entre pais e filhos e da percepção do conflito interparental na predição da DI em jovens. Esses resultados corroboram estudos internacionais, os quais apontam que as relações familiares conflituosas predizem a DI em jovens (Li et al., 2014; Yen et al., 2007). Além disso, ainda que participantes deste estudo estejam na fase adulta jovem, com idade média de cerca de 20 anos, percebe-se que as relações familiares representam uma parcela importante na predição de DI, mesmo nessa faixa etária.

Chama a atenção que os conflitos com os pais sobre a internet preponderam entre as variáveis familiares estudadas e talvez isso repercuta na relação conjugal dos pais, o que pode ser explicado pela sensação de ameaça do conflito interparental, expressa entre os preditores da DI. Pode-se pensar que talvez isso ocorra devido à posição do pai nos cuidados com os filhos (Wagner et al., 2002; Wagner et al., 2005). Em famílias tradicionais, caso deste estudo, no qual $60 \%$ são de famílias nucleares, onde o pai ainda é mais responsável pelos ingressos econômicos e a mãe pelos cuidados com a prole; o pai pode ter menor habilidade para lidar com os problemas referentes ao cotidiano dos filhos, tornando os conflitos mais intensos e deletérios.

A partir desse entendimento, considerando-se as diferenças entre pai e mãe no relacionamento com os filhos (Bögels \& Perotti, 2014), hipotetiza-se que o pai pode entrar em conflito com o filho utilizando-se de uma abordagem mais coercitiva, uma vez que as brigas com a mãe parecem não ser efetivas na resolução do problema, ou seja, a diminuição do uso de internet. Isso pode explicar por que os conflitos com o pai preponderam sobre os da mãe na predição da DI, mesmo que os conflitos com ela sejam mais frequentes. Sendo assim, essa forma inadequada do pai de lidar com o problema, segundo a ótica da mãe, faz com que ela também mantenha os conflitos sobre a internet com o filho na tentativa de resolver o problema e protege-lo dos desentendimentos com o pai (Bögels \& Perotti, 2014). No entanto, essas diferentes formas de lidar com as discórdias familiares fazem com que o filho tenha respostas negativas à conduta dos pais (Bolsoni-Silva et al., 2009) ao utilizar ainda mais a internet como forma de se isolar dessas dificuldades familiares (Young \& Abreu, 2011). Essa emaranhada teia de desentendimentos pode explicar a natureza sistêmica dos conflitos familiares (Erel
\& Burman, 1995) em torno da internet e sugerir que a família como um todo deve ser considerada quando se pensa na prevenção de DI entre os jovens.

Tendo em vista a dinâmica de relações conflituosas sobre a internet que pode envolver a família, o apoio emocional paterno aparece como um preditor negativo para a DI em jovens. Diante do papel relevante do pai no processo de socialização dos filhos (Bögels \& Perotti, 2014), pode-se pensar que a prática de apoio emocional, a qual se caracteriza por maior proximidade afetiva, disponibilidade e interesse pelo filho (Teixeira et al., 2006), pode atuar como um fator de proteção para os problemas de ajustamento que apresentam, entre suas características, as dificuldades de socialização.

\section{Considerações Finais}

Este estudo buscou analisar o efeito preditor dos motivos de conflito entre pais e filhos, do conflito interparental e das práticas educativas parentais para a dependência de internet em uma amostra de jovens brasileiros. Os principais preditores explicaram 21,2\% da DI. Destaca-se que esse percentual é um indicador importante de que as variáveis familiares investigadas têm um efeito considerável na predição da DI tendo em vista que esse é um fenômeno complexo e de natureza multifatorial. Sendo assim, sugere-se que estratégias para a prevenção da DI possam ser organizadas considerando não só a população jovem, mas tendo como um dos objetivos instrumentalizar os pais para lidarem com problemas relacionados à internet.

Pode-se perceber que, embora a idade média dos participantes tenha sido de cerca de 20 anos, caracterizando-se pela fase adulta jovem, os conflitos entre pais e filhos aparecem como preditores de DI. Além disso, a prática de apoio emocional paterno se mostrou como um fator de proteção, ainda nessa faixa etária.

Ressalta-se que os dados encontrados são relevantes para o contexto clínico, uma vez que podem servir como embasamento empírico para a prática dos profissionais que atendem casos de DI, os quais podem enfocar que a prática educativa de controle excessivo da internet aliada a desentendimentos familiares por esse motivo não diminui a DI em jovens, ao contrário, fomenta. Ademais, é necessário considerar que esses resultados estão embasados na percepção dos jovens, os quais podem perceber o controle por parte dos pais como algo negativo. 
Cabe sinalizar que esse trabalho apresenta, naturalmente, algumas limitações. Entre elas, está a escolha da amostra dos menores de idade pelo critério de conveniência, a idade média e o nível de escolaridade dos participantes. Aliado a isso, está o fato de que nenhum participante foi classificado como usuário grave, conforme critérios do IAT. Dessa forma, essas considerações são necessárias, uma vez que podem ter influenciado nos resultados obtidos, entre eles as baixas correlações da DI com as variáveis estudadas.

Dentro dessa perspectiva, salienta-se que esses resultados apresentam um panorama interessante $\mathrm{e}$ ainda pouco explorado da DI no contexto nacional, os quais se assemelham aos resultados encontrados em pesquisas realizadas com jovens de outros países. Entretanto, esses achados indicam que há espaço para outras pesquisas, destacando-se os delineamentos qualitativos, que busquem compreender com maior profundidade as relações familiares, de um modo geral, em torno da internet. Ademais, estudos com outras faixas etárias poderiam indicar as repercussões das relações familiares no uso de internet em longo prazo.

\section{Referências}

Abreu, C. N., Karam, R. G., Góes, D. S., \& Spritzer, D. T. (2008). Dependência de internet e de jogos eletrônicos: Uma revisão. Revista Brasileira de Psiquiatria, 30(2), 156-167. doi: 10.1590/ S1516-44462008000200014

Ak, Ş., Koruklu, N., \& Yilmaz, Y. (2013). A study on turkish adolescent's internet use: Possible predictors of internet addiction. Cyberpsychology, Behavior, and Social Networking, 16(3), 205-209. doi:10.1089/ cyber.2012.0255

Benetti, S. P. C. (2006). Conflito conjugal: Impacto no desenvolvimento psicológico da criança e do adolescente. Psicologia: Reflexão e Crítica, 19(2), 261-268. doi: 10.1590/S0102-79722006000200012

Bernal, A. C. L. (2012). Funcionamiento familiar, conflictos con los padres y satisfacción con la vida de familia en adolescentes bachilleres. Acta Colombiana de Psicología, 15(1), 77-85.

Bögels, S. M., \& Perotti, E. C. (2014). Does father know best? A formal model of the paternal influence on childhood social anxiety. Journal of Child and Family Studies, 20(2), 171-181. doi: 10.1007/ s10826-010-9441-0
Bolsoni-Silva, A. T., Paiva, M. M., \& Barbosa, C. G. (2009). Problemas de comportamento de crianças/ adolescentes e dificuldades de pais/cuidadores: Um estudo de caracterização. Psicologia Clínica, 21, 169-184. doi: 10.1590/S0103-56652009000100012

Buehler, C., \& Gerard, J. M. (2002). Marital conflict, ineffective parenting, and children's and adolescents' maladjustment. Journal of Marriage and Family, 64(1), 78-92. doi: 10.1111/j.1741-3737.2002.00078.x

Centro de Estudos sobre as Tecnologias da Informação e Comunicação [CETIC]. (2012). Pesquisa TIC Kids On-line Brasil. Recuperado de thttp://www. cetic.br/usuarios/kidsonline /2012/criancas.htm irado de

Conti, M. A., Jardim, A. P., Hearst, N., Cordás, T. A., Tavares, H. \& Abreu, C. N. (2012). Avaliação da equivalência semântica e consistência interna de uma versão em português do Internet Addiction Test (IAT). Revista de Psiquiatria Clínica, 39(3), 106-110. doi: 10.1590/S0101-60832012000300007

Dancey, C. P., \& Reidy, J. (2006). Estatística sem matemática para psicologia: Usando SPSS para Windows. ( $3^{\mathrm{a}}$ ed.). Porto Alegre: Artmed.

Dell’Osso, B., Altamura, A. C., Allen, A., Marazziti, D., \& Hollander, E. (2006). Epidemiologic and clinical updates on impulse control disorders: A critical review. European Archives of Psychiatry and Clinical Neuroscience, 256(8), 464-475. doi: 10.1007/ s00406-006-0668-0

Erel, O., \& Burman, B. (1995). Interrelatedness of marital relations and parent-child relations: $A$ meta-analytic review. Psychological Bulletin, 118(1), 108-132. doi: 10.1037/0033-2909.118.1.108

Fortson, B. L., Scotti, J. R., Chen,Y. C., Malone, J., \& Del Ben. (2007). Internet use, abuse, and dependence among students at a southeastern regional university. Journal of American College Health, 56(2), 137-144. doi: 10.3200/JACH.56.2.137-146

Goulart, V. R., Wagner, A., \& Mosmann, C. P. (2014). Os filhos e os conflitos conjugais: Revisando os modelos e os achados empiricos. Manuscrito submetido para publicação.

Grych, J. H., Seid, M. \& Fincham, F. D. (1992). Assessing marital conflict from the child's perspective: The children's perception of interparental conflict 
scale. Child Development, 63, 558-572. doi: 10.1111/ j.1467-8624.1992.tb01646.x

Jelenchick, L. A., Megan, T. B., \& Moreno, A. (2012). Assessing the psychometric properties of the Internet Addiction Test (IAT) in US college students. Psychiatry Research, 196(2-3), 296-301. doi: 10.1016/j.psychres.2011.09.007

Lam, L. T., Peng, Z. W., Mai, J. C., \& Jing, J. (2009). factors associated with internet addiction among adolescents. Cyberpsychology \& behavior, 12(5), 551555. doi: 10.1089/cpb.2009.0036

Li, W., Garland, E. L., \& Howard, M. O. (2014). Family factors in Internet addiction among Chinese youth: A review of English- and Chinese-language studies. Computers in Human Behavior, 31(0), 393411. doi:10.1016/j.chb.2013.11.004

Linares, M. C. G., Rusillo, M. T. C., Cruz, M. J. D. L. T., Fernández, M. D. L. V. C., \& Arias, P. F. C. (2011). Prácticas educativas paternas y problemas internalizantes y externalizantes en adolescentes españoles. Psicothema, 23(4), 654-659.

Liu, W. M., Ali, S. R., Soleck, G., Hopps, J., \& Pickett Jr, T. (2004). Using social class in counseling psychology research. Journal of Counseling Psychology, 51(1), 3. doi: 10.1037/0022-0167.51.1.3

Mosmann C. P., Costa, C., Luz, S. K., \& Silva, A. M. (2014). Filhos com sintomas psicológicos clínicos: Papel discriminante da conjugalidade, coparentalidade e parentalidade. Manuscrito submetido para publicação.

Mosmann, C. P. (2007). A Qualidade Conjugal e os Estilos Educativos Parentais. (Tese de Doutorado). Recuperado de http://repositorio.pucrs.br/dspace/ handle/10923/4768

Mosmann, C. P., Wagner, A., \& Sarriera, J. (2008). A qualidade conjugal como preditora dos estilos educativos parentais: $\mathrm{O}$ perfil discriminante de casais com filhos adolescentes. Psicologia, 22(2), 161-182.

Núcleo de Informação e Coordenação do Ponto BR [NICBR]. (2012). Pesquisa sobre o uso das tecnologias de informação e comunicação no Brasil: TIC Domicílios e TIC Usuários 2012. São Paulo: Comitê Gestor da Internet no Brasil. Recuperado de http://www.cetic.br/ usuarios/tic/2012/index.htm

Park, S. K., Kim, J. Y., \& Cho, C. B. (2007). Prevalence of Internet addiction and correlations with family factors among South Korean adolescents. Adolescence, 43(172), 895-909.

Teixeira, M. A. P., Oliveira, A. M., \& Wottrich, S. H. (2006). Escalas de Práticas Parentais (EPP): Avaliando dimensões de práticas parentais em relação a adolescentes. Psicologia: Reflexão e Crítica, 19(3), 433-441. doi: 10.1590/S0102-79722006000300012

Villas-Boas, A. C. V. B., Dessen, M. A., \& Melchiori, L. E. (2010). Conflitos conjugais e seus efeitos sobre o comportamento de crianças: Uma revisão teórica. Arquivos Brasileiros de Psicologia, 62, 91-102.

Wagner, A. (2010). Conjugalidade e parentalidade: Estratégias de resolução de conflitos de pais e filhos (Manuscrito não publicado). Universidade Federal do Rio Grande do Sul, Porto Alegre.

Wagner, A., Falcke, D., Silveira, L. M. O., \& Mosmann, C. P. (2002). A comunicação em famílias com filhos adolescentes. Psicologia em Estudo, 7(1), 75-80. doi: 10.1590/S1413-73722002000100010

Wagner, A., Predebon, J., Mosmann, C., \& Verza, F. (2005). Compartilhar tarefas? Papéis e funções de pai e mãe na família contemporânea. Psicologia: Teoria e Pesquisa, 21(2), 181-186. doi: 10.1590/ S0102-37722005000200008

Weinstein, A., \& Lejoyeux, M. (2010). Internet addiction or excessive internet use. The American Journal of Drug and Alcobol Abuse, 36, 277-283. doi: 10.3109/00952990.2010.491880

Widyanto, L., \& McMurran, M. (2004). The psychometric properties of the Internet Addiction Test. CyberPsychology and Behavior, 7(4), 443-450. doi: 10.1089/cpb.2004.7.443

Yen, J. Y., Yen, C. F., Chen, C. C., Chen, S. H., \& Ko, C. H. (2007). Family factors of Internet addiction and substance use experience in Taiwanese adolescents. CyberPsychology and Behavior, 10(3), 323-329. doi:10.1089/cpb.2006.9948

Young, K.S. (1996). Addictive use of the internet: A case that breaks the stereotype. Psychological Reports, 79, 899-902.

Young, K. S. (1998). Internet addiction: the emergence of a new clinical disorder. CyberPsychology and Behavior, 1(3), 237-244. 
Young, K. S., \& Abreu, C. N. (Orgs.). (2011). Dependência de internet: Manual e guia de avaliação e tratamento. Porto Alegre: Artmed.

Yu, L., \& Shek, D. T. L. (2013). Internet addiction in Hong Kong adolescents: A three-year longitudinal study. Journal of Pediatric and Adolescent Gynecology, 26(3), S10-S17. doi:10.1016/j.jpag.2013.03.010

Recebido 25/08/2015

Reformulado 08/10/2015

Aceito 27/10/2015

Sobre as autoras:

Michele Terres-Trindade é psicóloga, mestre em Psicologia Clínica pela Universidade do Vale do Rio dos Sinos e professora do curso de graduação em Psicologia da Universidade Feevale.

E-mail:micheleterres@hotmail.com

Clarisse Pereira Mosmann é psicóloga, doutora em Psicologia pela Pontifícia Universidade Católica do Rio Grande do Sul e professora do curso de graduação e do programa de pós-graduação em Psicologia da Universidade do Vale do Rio dos Sinos.

E-mail: clarissepm@unisinos.br

Contato com as autoras:

Av. Iguaçu, 119/405, Petrópolis

CEP: 90470-430

Porto Alegre-RS, Brasil 